VOL. 8 (1973), 23-26.

\title{
Uniqueness and nonuniqueness in mean boundary value problems
}

\section{Chin-Hung Ching and Charles $K$ Chui}

We give some sufficient conditions to guarantee the uniqueness of certain mean boundary value problems for a circle. Also we show that, in general, we cannot expect uniqueness of the problem for an arc unless the function is analytic in a neighborhood of the unit circle or some shifted means of the function are also known.

Let $U$ denote the open unit disc and $T$ the unit circle. For a function $f$ continuous on an arc $\left\{e^{i 2 \pi t}, t_{1} \leq t \leq t_{2}\right\}$ of $T$, we consider the arithmetic means,

$$
\begin{aligned}
& \boldsymbol{s}_{n}\left(f ; t_{1}, t_{2}\right)=\frac{1}{n} \sum_{k=1}^{n} f\left(e^{i 2 \pi k\left(t_{2}-t_{1}\right) / n+i 2 \pi t_{1}}\right), \\
& \tilde{s}_{n}\left(f ; t_{1}, t_{2}\right)=\frac{f\left(e^{i 2 \pi t_{1}}\right)+f\left(e^{i 2 \pi t_{2}}\right)}{2 n}+\frac{1}{n} \sum_{k=1}^{n-1} f\left(e^{i 2 \pi k\left(t_{2}-t_{1}\right) / n+i 2 \pi t_{1}}\right),
\end{aligned}
$$

$n=1,2, \ldots$, and the limit,

$$
\begin{aligned}
s_{\infty}\left(f ; t_{1}, t_{2}\right) & =\lim _{n \rightarrow \infty} s_{n}\left(f ; t_{1}, t_{2}\right) \\
& =\lim _{n \rightarrow \infty} \tilde{s}_{n}\left(f ; t_{1}, t_{2}\right) .
\end{aligned}
$$

Then $s_{n}(f ; 0,1)=\tilde{s}_{n}(f ; 0,1)$ for all $n$. As usual, let If $^{p}$ be the Hardy spaces and $A$ be the space of functions holomorphic in $U$ and

Received 8 August 1972. 
continuous on $\bar{U}$. We have the following results.

THEOREM 1. Let $f(z)=\sum a_{n} z^{n}$ be in $A$ and satisfy $s_{n}(f ; 0,1)=0$ for all $n$. Then $f$ is the zero fronction if one of the following conditions is satisfied:

(i) $a_{n}=0\left(\frac{1}{n^{1+\varepsilon}}\right)$ for some $\varepsilon>0$;

(ii) $\sum_{k=N}^{\infty}\left|a_{k}\right|=0\left(\frac{1}{N}\right)$;

(iii) there exists a prime $q>0$ such that $a_{k}=0$ if $k \neq q^{m}$ for some integer $m$;

(iv) $f^{\prime}$ belongs to $H^{p}$ for some $p, I<p \leq \infty$.

By similar methods we can conclude that $f$ is determined by the means

$$
\frac{1}{n} \sum_{k=1}^{n} f\left(z_{n, k}\right)
$$

with $z_{n, k}=\rho\left(e^{i 2 \pi k / n}\right)$ for any diffeomorphism $\rho$ of $T$ satisfying $\overline{\rho\left(e^{i \theta}\right)}=\rho\left(e^{-i \theta}\right)$.

We remark that there exist polynomials $p_{m}$ with ${ }_{n}\left(p_{m} ; 0,1\right)=\delta_{m, n}$ (cf. [1]) and that if $\sum\left|s_{n}^{-s_{\infty}}\right| n^{\varepsilon}$ converges for some $\varepsilon>0$ and one of the above four conditions holds, we can use results in [1] to obtain an explicit formula to recapture $f$ from its means $s_{n}(f ; 0,1)$. However, for a proper subarc $K=\left\{e^{i 2 \pi t}: t_{1} \leq t \leq t_{2}, 0<t_{2}-t_{1}<1\right\}$, we can construct a nonzero function $f$ in $A$, infinitely differentiable relative to $\bar{U}$, holomorphic in a neighborhood of $K$, and $s_{n}\left(f ; t_{1}, t_{2}\right)=\tilde{s}_{n}\left(f ; t_{1}, t_{2}\right)=0$ for all $n=1,2, \ldots$. On the other hand, we have uniqueness for an arc if certain extra conditions are satisfied, namely,

THEOREM 2. Let $f$ be in $A$ such that either $\tilde{s}_{n}(f ; 0, \delta)=0$. or 
$\boldsymbol{s}_{n}(f ; 0, \delta)=0$ for all $n=1,2, \ldots$. Then $f$ is the zero function if one of the following conditions holds:

(i) $f$ is holomorphic in a neighborhood of the closed ronit disk;

(ii) $f^{\prime} \in H^{p}$ for $1<p \leq \infty$, and

$$
\begin{aligned}
& t_{n}(f ; 0, \delta)=\frac{f\left(e^{i 2 \pi \delta}\right)}{2 n}+\frac{1}{n} \sum_{k=1}^{n-1} f\left(e^{i 2 \pi \delta(2 k-1) /(2 n-1)}\right)=0, \\
& \text { for } n=1,2, \ldots .
\end{aligned}
$$

We note that combining Theorem 2 and the results of [1] and [2], we can obtain an explicit formula to recapture a $c^{l+\varepsilon}$ function $f$ if its means $\boldsymbol{s}_{n}, t_{n}$ are known on an arc, namely,

$$
f(z)=\lim _{\lambda \rightarrow \infty} \lambda h_{\lambda}(z) \int_{0}^{\delta} \frac{\overline{h_{\lambda}}\left(e^{i 2 \pi t}\right) g\left(e^{i 2 \pi t}\right)}{1-z e^{-i 2 \pi t}} d t
$$

with

$$
\begin{aligned}
g\left(e^{i 2 \pi t}\right)= & \sum_{m=1}^{\infty}\left[\tilde{s}_{2 m}(f ; 0, \delta)-\varepsilon_{\infty}(f ; 0, \delta)\right] p_{2 m}\left(e^{i 2 \pi t / \delta}\right) \\
& +\sum_{m=1}^{\infty}\left[\frac{2 m}{2 m-1} t_{m}(f ; 0, \delta)-s_{\infty}(f ; 0, \delta)\right] p_{2 m-1}\left(e^{i 2 \pi t / \delta}\right)+s_{\infty}(f ; 0, \delta)
\end{aligned}
$$

and

$$
h_{\lambda}(z)=\exp \left\{\frac{-\log (1+\lambda)}{2} \int_{0}^{\delta} \frac{1+z e^{-i 2 \pi t}}{1-z e^{-2 \pi t}} d t\right\} .
$$

Here

$$
p_{n}(z)=\sum_{k \uparrow n} \mu\left(\frac{n}{k}\right) z^{k}
$$

as in [1]. If $f$ is holomorphic in a neighborhood of $\bar{U}$ the formula for $g$ is a little simpler. The details of the proofs and related results will appear elsewhere. 


\section{References}

[1] Chin-Hung Ching and Charles K. Chui, "Representation of a function in terms of its mean boundary values", Bull. Austral. Math. Soc. 7 (1972), 425-427.

[2] D.J. Patil, "Representation of $H^{p}$ functions", Bull. Amer. Math. Soc. $78(1972), \cdot 617-620$.

Department of- Mathematics,

Texas A\&M University,

College Station,

Texas,

USA. 